\title{
Complementarity and redundancy of interactions enhance attack rates and spatial stability in host-parasitoid food webs
}

\author{
Guadalupe Peralta, ${ }^{1,6}$ Carol M. Frost, ${ }^{1}$ Tatyana A. Rand, ${ }^{2}$ Raphael K. Didham, ${ }^{3,4}$ and Jason M. Tylianakis ${ }^{1,5}$ \\ ${ }^{1}$ School of Biological Sciences, University of Canterbury, Private Bag 4800, Christchurch 8140 New Zealand \\ ${ }^{2}$ USDA-ARS Northern Plains Agricultural Research Laboratory, Sidney, Montana 59270 USA \\ ${ }^{3}$ School of Animal Biology, University of Western Australia, 35 Stirling Highway, Crawley, Western Australia 6009 Australia \\ ${ }^{4}$ CSIRO Ecosystem Sciences, Centre for Environment and Life Sciences, Underwood Avenue, Floreat, \\ Western Australia 6014 Australia \\ ${ }^{5}$ Department of Life Sciences, Imperial College London, Silwood Park Campus, Buckhurst Road, Ascot, \\ Berkshire SL5 7PY United Kingdom
}

\begin{abstract}
Complementary resource use and redundancy of species that fulfill the same ecological role are two mechanisms that can respectively increase and stabilize process rates in ecosystems. For example, predator complementarity and redundancy can determine prey consumption rates and their stability, yet few studies take into account the multiple predator species attacking multiple prey at different rates in natural communities. Thus, it remains unclear whether these biodiversity mechanisms are important determinants of consumption in entire predator-prey assemblages, such that food-web interaction structure determines community-wide consumption and stability.

Here, we use empirical quantitative food webs to study the community-wide effects of functional complementarity and redundancy of consumers (parasitoids) on herbivore control in temperate forests. We find that complementarity in host resource use by parasitoids was a strong predictor of absolute parasitism rates at the community level and that redundancy in host-use patterns stabilized community-wide parasitism rates in space, but not through time. These effects can potentially explain previous contradictory results from predator diversity research. Phylogenetic diversity (measured using taxonomic distance) did not explain functional complementarity or parasitism rates, so could not serve as a surrogate measure for functional complementarity.

Our study shows that known mechanisms underpinning predator diversity effects on both functioning and stability can easily be extended to link food webs to ecosystem functioning.

Key words: biodiversity; ecosystem functioning; food-web structure; insurance hypothesis; niche partitioning; parasitism; phylogenetic species variability; spatial variability; temporal variability.
\end{abstract}

\section{INTRODUCTION}

The relationship between biodiversity, ecosystem functioning, and stability has a long history of research (May 1973, Hooper et al. 2005, Gravel et al. 2011b, Fenoglio et al. 2012). Species diversity is usually positively related with rates of ecosystem functions (Hooper et al. 2005), and it may also affect variability in functioning, which is particularly important for services that benefit humans (Daily et al. 2000, Díaz et al. 2006, Daily et al. 2009, Sekercioglu 2010). Although much of the initial work on biodiversity effects focused on plant communities (Balvanera et al. 2006, Cardinale et al. 2006), a growing body of research has considered trophic (e.g., predator-prey) interactions in a diversity-function context, with important implications for

Manuscript received 13 August 2013; revised 15 November 2013; accepted 18 December 2013. Corresponding Editor: J. A. Rosenheim.

${ }^{6}$ E-mail: gdlp.peralta@gmail.com biological control (Cardinale et al. 2003, Ives et al. 2005, Snyder et al. 2006, Straub et al. 2008).

The effect of biodiversity on prey attack rates seems to depend on the trophic level at which diversity is considered (Duffy 2002). For example, higher prey diversity has been related to lower predation rates (Montoya et al. 2006), while higher predator diversity has been related to increased predation (Cardinale et al. 2003, Ives et al. 2005, Snyder et al. 2006, Tylianakis et al. 2006, Fenoglio et al. 2012). To add complexity, changes in predator diversity can also affect prey diversity (Chase et al. 2002), and changes to prey diversity can even alter the effects of predator diversity on attack rates (Wilby and Orwin 2013), suggesting that studying biodiversity within discrete trophic levels might not be the best way to predict changes in functioning of complex ecosystems. Moreover, it has been observed that evenness among predators can also exert a strong effect on consumption (Crowder et al. 2010). Thus, ecosystem functioning seems to be the net result of interactions within and among trophic levels (Hillebrand and Cardinale 2004, Douglass et al. 2008, Thompson et 
al. 2012, Wilby and Orwin 2013) and the relative abundances of different species, rather than just interactions between individual species pairs.

By combining information on predator and prey diversity and composition with measures of energy flow, indirect effects, and the frequency of interactions, food webs provide a natural tool with which to examine the effects of biodiversity on ecosystem functioning and stability (Thompson et al. 2012, Poisot et al. 2013). However, despite the clear potential for a link between trophic complexity (i.e., food-web structure) and consumption rates (Thompson et al. 2012), there has been little research examining functional outcomes in a foodweb context (Montoya et al. 2003, Macfadyen et al. 2009, Poisot et al. 2013), with most studies considering assemblages that only contain between two to six predator species. A few exceptions have extended the traditional biodiversity-functioning approach by considering the complexity of interactions among species and found important effects of the strength (Rooney and McCann 2012) and number of interactions (Montoya et al. 2003, Thébault and Loreau 2003) on prey consumption. In fact, it has even been suggested that the link between biodiversity and ecosystem functioning may have more to do with how species interact, i.e., the structure of food webs, than how many species form the community (Montoya et al. 2003, Thompson et al. 2012).

Recent theoretical work has suggested that resource complementarity (also termed functional complementarity) within food webs may affect ecosystem functioning (Poisot et al. 2013), with complementarity occurring when differences in resource-use strategies and functional traits allow species to specialize on different resources or on the same resource but in different locations or times (Hooper et al. 2005, Yachi and Loreau 2007, Richards and Schmidt 2010). Hence, functional complementarity can be understood as a community-level descriptor, which increases when species at the same trophic level differ from each other in the resources they use. Moreover, interaction patterns within food webs could also inform about redundancy in resource use, e.g., a number of species feeding on the same resource in the same way. This perresource species redundancy in consumers should, according to the insurance hypothesis, minimize variability in ecological functions (Yachi and Loreau 1999) by maintaining functioning when some consumer species decline in abundance (Walker 1992, Naeem and Li 1997). Similarly, spatial insurance effects may reduce site-to-site variability in functional rates (Loreau et al. 2003), yet these insurance effects have not to our knowledge been tested in diverse, natural terrestrial communities.

One reason for this lack of research could be that determining the trophic niche of many species of generalist predators under field conditions can be logistically difficult. However, with relatively special- ized predators, such as parasitoids, it becomes easier to quantify complementarity and redundancy in their trophic niches. Given that parasitoids make a strong contribution to the ecosystem service of pest control (Mills and Wajnberg 2008, Pennisi 2010), hostparasitoid communities provide avenues for developing and improving management strategies against herbivore pests (Tylianakis and Binzer, in press). Studies on host-parasitoid food webs have found parasitoid species richness to relate to increased parasitism rates and also temporal stability of parasitism (Cardinale et al. 2003, Tylianakis et al. 2006, Macfadyen et al. 2011), while others have found no effect (Marino and Landis 1996, Menalled et al. 1999, Macfadyen et al. 2011). These conflicting results may not be surprising, as theory suggests that the strength of any predator diversity effect depends on the extent to which different species partition their prey resources (Finke and Snyder 2008), which in turn can depend on predator trophic specialization and the diversity and distribution of prey (Tylianakis et al. 2008, Wilby and Orwin 2013). Thus, the way in which consumer interactions are structured in a food web could provide a link between predator diversity, prey resource partitioning, and their joint impact on attack rates and their variability.

Finally, because selection pressures associated with niche overlap can influence species diversification (Kozak and Wiens 2010), the phylogenetic diversity of a community may be a measure of its potential for resource complementarity (Maherali and Klironomos 2007). If this is the case, then predator relatedness across a community could be as informative as resource complementarity in explaining variation in attack rates. Moreover, measuring predator relatedness from samples of the community would be considerably easier, without needing to quantify predator-prey trophic links.

Here we use empirical host-parasitoid food webs from temperate native and plantation forests to test the following hypotheses. (1) Higher functional complementarity (i.e., host-resource partitioning among parasitoid species) will increase parasitism rates across the entire food web. (2) Resource overlap (redundancy) among parasitoid species that attack the same host species will provide insurance effects, leading to lower variability (i.e., higher stability) of parasitism rates through time and space. Consequently, communities dominated by generalist parasitoid species should have more spatially and temporally stable attack rates than communities with fewer generalists. (3) Because niche partitioning promotes species coexistence and diversification as well as complementarity (Kozak and Wiens 2010, Poisot et al. 2013), we hypothesize that phylogenetic diversity of parasitoids within a community will be positively correlated with functional complementarity in host use and, as a consequence, parasitism rates on hosts. 


\section{Methods}

Study region

In the Nelson-Marlborough region of New Zealand $\left(172^{\circ} 47^{\prime}\right.$ to $173^{\circ} 53^{\prime} \mathrm{E}$ and $41^{\circ} 12^{\prime}$ to $\left.41^{\circ} 33^{\prime} \mathrm{S}\right)$, we sampled host-parasitoid food webs at eight different sites (with a minimum distance between sites of at least $2.7 \mathrm{~km}$ ). Each site was composed of two adjacent forest types representative of the two predominant forest types in the region (native southern beech and exotic pine plantation). At each site we established four sampling plots, with two in each forest type, one at the edge adjacent to the other forest type, and one at the forest interior (for more details on the study region, see Appendix A). In total, therefore, we had 32 sampling plots, but given that the four plots from each site were non-independent, we nested sampling plots within sites in all the analyses. Even though there was high variability in food-web structure and absolute parasitism rates among sampling plots, there were no significant differences in structure or function between forest types or locations (edge vs. interior) of the sampling plots within forest types, therefore, we do not discuss habitat type differences here. Moreover, any differences among habitat types would simply contribute to the error variance of our analyses and make it less likely to detect a significant effect of food-web structure on parasitism rates across all plots.

\section{Sampling}

In each sampling plot, we collected Lepidoptera larvae (herbivores, also referred to as hosts) along a 50 $\times 2 \mathrm{~m}$ transect by beating all the vegetation up to a height of $2 \mathrm{~m}$ with a $1 \mathrm{~m}$ long PVC pipe over white sheets $\left(1 \times 1 \mathrm{~m}^{2}\right)$ placed under the plants to collect the dislodged herbivores. In addition, at 5-m intervals along each transect (i.e., at 10 sampling points) we sampled the canopy of the nearest accessible tree by using a 9-m pole pruner to cut subcanopy branches, which were then beaten over the sheets. We sampled each plot once per month from December to February (2009-2010) and November to February (2010-2011). For more details on the sampling procedure, see Appendix A.

After herbivore hosts were collected in the field, we moved them to the laboratory for identification and reared them until either they developed into adults or parasitoids emerged. Each herbivore host was reared individually in a separate container under controlled conditions $\left(16^{\circ} \mathrm{C}\right.$, relative humidity of $60 \%$, and a light rhythm of 16L:8D) and fed with leaves of the plant species from which it had been collected, plus a supplemental general Lepidoptera growth medium (beet army worm diet from Bio-Serv Entomology Custom Research Diets and Environmental Enrichment Products, Frenchtown, New Jersey, USA). Parasitoids were identified by their morphology and using molecular barcoding when species-level identification was not possible, for example, with males where taxonomic keys apply only to females or with cryptic species (see Appendix A for more details on molecular identification techniques). A list of the host and parasitoid species involved in this study and locations where voucher specimens have been deposited can be found in Appendix A.

\section{Analyses}

We used a quantitative food-web approach to link parasitoids with their hosts (Tylianakis et al. 2007). The weighting (strength) of trophic links was measured as interaction frequency, which comprises an estimate of energy flow. First, we tested the effects of functional complementarity on parasitism rates. We calculated cumulative parasitism rates (parasitism rates across the entire sampling period) for each sampling plot as the number of parasitoids that emerged divided by the total number of herbivore hosts collected over the total sampling period. Hosts that died for any reason before emerging as an adult moth or parasitoid were not included in this denominator, as we could not be certain that these were not parasitized. We measured parasitoid functional complementarity as the total branch length of a dendrogram based on quantitative differences among parasitoids in their resource use, i.e., host species consumed (Devoto et al. 2012). That is, we first created one matrix for each sampling plot in which host species were represented as rows, parasitoid species as columns, and the values within the cells represented the frequency with which each host-parasitoid interaction occurred. Because the functional complementarity metric was initially highly correlated with parasitoid abundance (Appendix B), we standardized the host-parasitoid matrices by dividing the values of each cell by the total number of parasitoids present in that food web. We then created a (Euclidean) distance matrix among parasitoid species from each of the standardized host-parasitoid matrices, by using the fd function of the Bipartite package (Dormann et al. 2008) for the R environment (R Development Core Team 2012). Values within this distance matrix represent the similarity among parasitoid species regarding the resources they shared. In this sense, it is analogous to the distance matrix of species composition across sites used in community ordinations, but in this case, we measured distances in host composition across parasitoid species. Then, from the distance matrix, parasitoid species were clustered according to their similarities in host resource use and a dendrogram was created, with distances among parasitoid species (branches of the dendrogram) equivalent to their dissimilarity in resource use (i.e., the longer the branch length among two parasitoid species, the fewer the resources they share). Finally, the total branch length of the dendrogram was calculated, which represents the total functional complementarity of the web, after controlling for differences in parasitoid abundance among webs. The highest functional complementarity would therefore arise when predator 
species are specialized on different resources, while a community formed by generalist predators that share resources among them would have lower functional complementarity. By adopting a community-wide metric of functional complementarity that takes into account the feeding niche of all the species within a community, the overlap in resource use among species can be delimited with more precision and related to community-wide measures of consumption.

We tested the effect of parasitoid functional complementarity, as a fixed predictor, on cumulative parasitism rates (the response variable) using a generalized linear mixed-effects model (GLMM) with a binomial error distribution and the canonical logit link function. We included sampling plots nested within sites as random factors to control for the non-independence of plots within sites. Parameter estimates for fixed effects in the minimal models were tested for significance using a Wald's $Z$ test (Bolker et al. 2009).

Because it is possible that host niche partitioning among parasitoids could be associated with parasitoid speciation (Kozak and Wiens 2010), communities with more distantly related parasitoids might be expected to have the greatest complementarity, and thus phylogenetic diversity might be a useful proxy for complementarity. To test for this possibility, we included parasitoid phylogenetic diversity as a predictor in the model, calculated as phylogenetic species variability (Helmus et al. 2007) with the psd function of the picante $\mathrm{R}$ package (Kembel et al. 2010). To determine phylogenetic species variability, we constructed phylogenetic trees based on taxonomic distances (Poulin and Mouillot 2003) among parasitoid species. Taxonomic distances reflect the number of steps that separate each pair of species, where steps can be defined according to the Linnaean classification, including order, suborder, superfamily, family, genus, and species, following Cagnolo et al. (2011). Even though using taxonomic distance has the disadvantage that it underestimates evolutionary differences (Weiblen et al. 2006), it has the advantage of being easily used for any food web where species have been identified and can be used for poorly studied taxa for which phylogenies are difficult to obtain. The phylogenetic species variability index can acquire values between 0 and 1 , increasing toward 1 when species within a community are less phylogenetically related (Helmus et al. 2007), i.e., when phylogenetic diversity is higher. Because we used taxonomic distances for calculating this metric, we refer to it hereafter as taxonomic diversity, although it takes into account species relatedness.

Second, we tested the effect of functional redundancy on variability in parasitism rates in both space and time. Temporal variability in parasitism rates in each sampling plot across sampling months was estimated as the standard deviation in parasitism rates across months divided by the mean parasitism rate in that sampling plot, i.e., the coefficient of variation (CV) (Tylianakis et al. 2006). As a measure of spatial variability of parasitism rates across sites, we calculated the $\mathrm{CV}$ of parasitism rates among sampling plots within a site (standard deviation of parasitism rates across sampling plots divided by the mean parasitism rate in that site). We defined parasitoid functional redundancy as the average number of parasitoid species attacking each host species weighted by the frequency of the trophic interactions, otherwise known as food-web quantitative vulnerability (Bersier et al. 2002), calculated at the sampling plot and at the site levels, using the networklevel function (Dormann et al. 2009) from the $R$ Bipartite package (Dormann et al. 2008). Hence, the more parasitoid species attacking each host species, the higher the parasitoid functional redundancy.

To evaluate the variability in parasitism rates through time, we used a GLMM with a Gaussian error distribution. We used a Markov chain Monte Carlo (MCMC) resampling procedure to estimate $P$ values, rather than an $F$ test to avoid problems associated with estimating degrees of freedom (Bolker et al. 2009). We entered temporal variability $(\mathrm{CV})$ of parasitism rates as the response variable, parasitoid redundancy (calculated at the sampling plot level) as the predictor, and sampling plots nested within sites as random factors. To evaluate the variability in parasitism rates through space, we used a general linear model (with a Gaussian error distribution), with spatial variability of parasitism rates (between plots within a site) as the response variable and parasitoid redundancy (calculated at the site level) as the predictor. Parameter estimates for fixed effects for this last model were tested for significance using a $t$ test (Bolker et al. 2009).

The effects of predator or parasitoid species richness on prey attack rates and stability have been tested previously (e.g., Cardinale et al. 2003, Ives et al. 2005, Snyder et al. 2006, Tylianakis et al. 2006, Macfadyen et al. 2011), and we were interested here in whether trophic complementarity and redundancy provide more information than does simple, and more easily measured, species richness. Therefore, we also incorporated, in all the models, host species richness, parasitoid species richness, and the weighted number of different interactions (quantitative connectance; Bersier et al. 2002) as covariates, so that we could test for effects of interaction structure on parasitism rates after accounting for the effects of the number of species in webs and the number of links between species.

Each model initially included all the variables mentioned above and was then simplified to the minimum adequate model by stepwise removal of fixed predictors until no further reduction in residual deviance (as measured by the Akaike information criteria, AIC) was observed (Bolker et al. 2009). We performed this model reduction process twice for each model to better understand the mechanisms underpinning effects of food-web structure on functioning and stability. The first time we did not allow removal of the covariates (parasitoid and host species richness and connectance) 

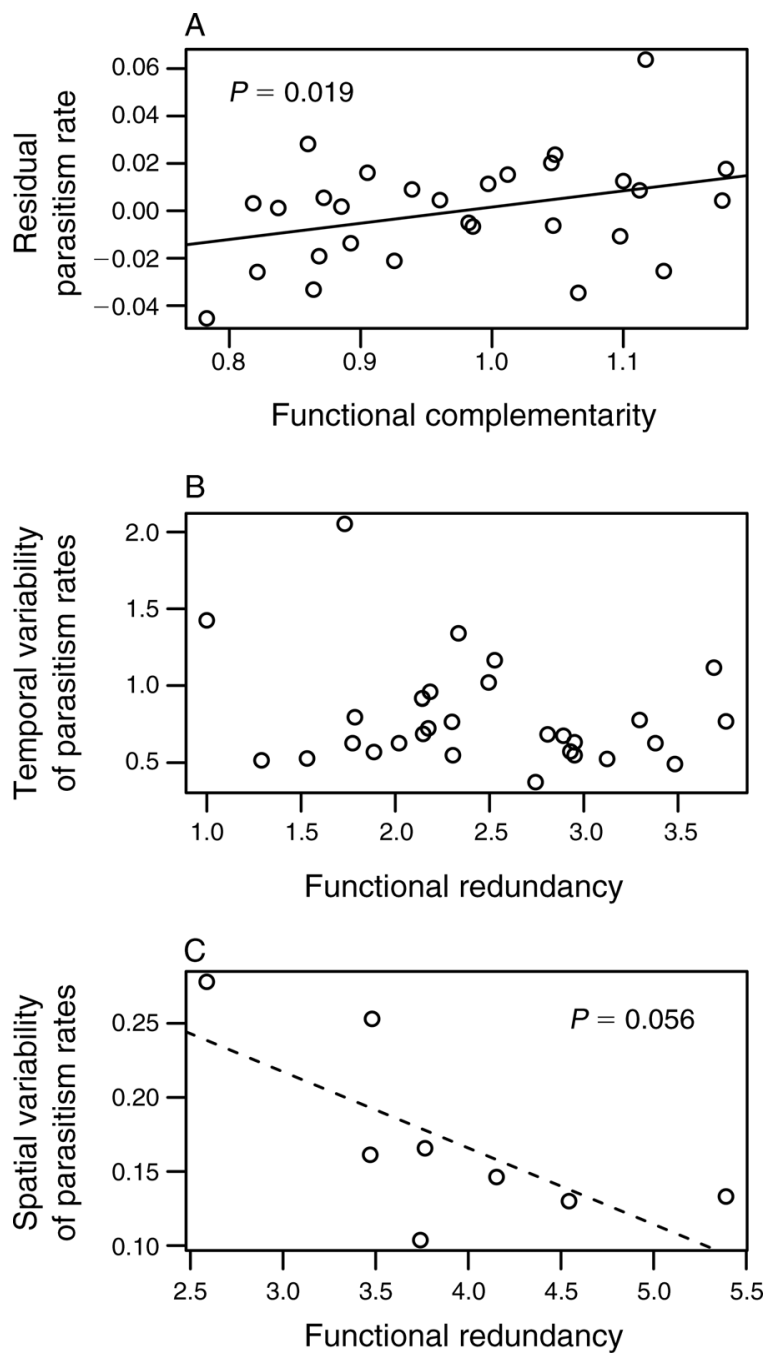

FIG. 1. Relationship between (A) cumulative parasitism rates (over the entire sampling period) and standardized parasitoid functional complementarity (showing the partial effect of complementarity in a model with parasitoid species richness), (B) temporal variability of parasitism rates and parasitoid functional redundancy, and (C) spatial variability of parasitism rates and parasitoid functional redundancy. Although generalized linear mixed-effects models were used to account for the non-independence of plots within sites (see Methods), the least-squares trend line is shown to illustrate the direction of effect. A solid line represents a significant relationship; a dashed line represents a marginally significant relationship.

during simplification in order to determine whether functional complementarity and redundancy provided any information over and above the known effects of diversity. The second time we allowed removal of covariates to test whether any functional complementarity and redundancy effects depended on the partial effects of the covariates (i.e., whether interaction pattern only mattered at a given level of diversity and connectance, but excluding these covariates generated too much noise to detect any effects of complementarity and redundancy). Furthermore, because spatial variability was calculated at the site level, and consequently had low power (8 replicates, giving 7 degrees of freedom), removal of covariates would reduce the probability of a Type II error and hence detect potential effects that the full model with all the covariates might have not detected due to low power.

All the analyses were conducted in the $\mathrm{R}$ 2.15.2 environment (R Development Core Team 2012). We used the lme4 package (Bates and Maechler 2009) for GLMMs and the languageR package pvals.fnc function (Baayen 2010) for the MCMC procedure in the temporal variability Gaussian models. We tested for outliers, and three of our 32 webs exhibited strong leverage, two for complementarity and one for redundancy. We therefore removed these from the analyses in order to avoid spurious trends, even though the results with or without the outliers did not change qualitatively. We also tested for overdispersion of residuals in the binomial models and for normality and homoscedasticity in the Gaussian models, which required the temporal coefficient of variation of parasitism rates to be $\log$ transformed to meet model assumptions.

\section{RESUlts}

Overall, we collected 5743 Lepidoptera larvae from 90 species (Appendix A), with an average parasitism rate of $12.0 \% \pm 3.6 \%$ [mean $\pm \mathrm{SD}$ ] across sampling plots. Parasitoids that emerged were from 61 different species (Appendix A). Sampling plots had on average $8 \pm 2$ host species and $10 \pm 3$ parasitoid species, with mean parasitoid taxonomic diversity of $0.636 \pm 0.091$.

After accounting for potential covariate effects of host species richness, parasitoid species richness, and connectance (number of different interactions weighted by their frequency of occurrence) in our GLMM, we still found that food webs with higher parasitoid functional complementarity had significantly higher cumulative parasitism rates $(Z=2.34, P=0.019$; Fig. $1 \mathrm{~A}$; see Appendix D). This effect was not dependent on the partial effects of host species richness and connectance, as complementarity in resource use still increased parasitism rates significantly if those covariates were excluded from the model $(Z=2.11, P=0.035$; Table 1). Neither host species richness $(Z=0.53, P=0.598)$ nor connectance $(Z=1.16, P=0.244)$ significantly affected parasitism rates (Appendix D), and these were not even retained in the best-fitting model when allowing for their removal (Table 1). Parasitoid species richness had a significant positive effect on parasitism rates both when accounting $(Z=3.30, P>0.001$; Appendix $\mathrm{D}) \operatorname{or} \operatorname{not}(Z$ $=3.35, P>0.001$; Table 1$)$ for differences in host species richness and connectance. Our measure of parasitoid taxonomic diversity (phylogenetic species variability) was not correlated with parasitoid functional complementarity $(r=0.01, P=0.953$; Appendix B) nor retained in the best-fitting model (Table 1), suggesting that 
TABLE 1. Coefficients for best-fitting GLMM testing the effect of standardized parasitoid functional complementarity and redundancy on parasitism rates and their stability respectively.

\begin{tabular}{|c|c|c|c|}
\hline Response variables and fixed effects & Estimate & $Z / t$ & $P / P_{\mathrm{MCMC}}$ \\
\hline \multicolumn{4}{|l|}{ Parasitism rates } \\
\hline Intercept & $-3.498 \pm 0.483$ & -7.243 & $>0.001$ \\
\hline Parasitoid richness & $0.054 \pm 0.016$ & 3.354 & $>0.001$ \\
\hline Standardized parasitoid functional complementarity & $0.861 \pm 0.408$ & 2.113 & 0.035 \\
\hline \multicolumn{4}{|l|}{ Temporal variability of parasitism rates } \\
\hline Intercept & $-0.714 \pm 0.223$ & -3.20 & 0.037 \\
\hline Connectance & $3.525 \pm 1.021$ & 3.45 & 0.009 \\
\hline Parasitoid functional redundancy & $-0.086 \pm 0.059$ & -1.44 & 0.164 \\
\hline \multicolumn{4}{|l|}{ Spatial variability of parasitism rates } \\
\hline Intercept & $0.372 \pm 0.086$ & 4.29 & 0.005 \\
\hline Parasitoid functional redundancy & $-0.051 \pm 0.022$ & -2.36 & $0.056 \dagger$ \\
\hline
\end{tabular}

Notes: Best-fitting model results, after allowing for covariate removal (host species richness, parasitoid species richness, and connectance), for models testing the effect of standardized parasitoid functional complementarity on cumulative parasitism rates (with binomial error, $Z$ test), connectance and parasitoid functional redundancy on the temporal variation $(\mathrm{CV})$ of parasitism rates (with Gaussian error, $t$ value, and $P$ values estimated by Markov chain Monte Carlo [MCMC] procedure), and parasitoid functional redundancy on spatial variation (CV) of parasitism rates (with Gaussian error, $t$ test). Estimates are given as mean \pm SE. Values in boldface type indicate significant results $(\alpha=0.05)$.

$\dagger$ Marginally significant result.

having more distantly related species does not guarantee functional complementarity among parasitoids.

Contrary to our expectations, parasitoid functional redundancy did not affect the temporal variability in parasitism rates (coefficient of variation of parasitism rates through time; $\left.t=-1.44, P_{\mathrm{MCMC}}=0.164\right)$, and this term was only retained in the model when allowing for covariate removal (Table 1, Fig. 1B). When preserving the covariates in the model selection procedure, parasitoid redundancy was not retained in the best-fitting model (Appendix D). In contrast, connectance was retained in the model whether covariates were preserved (Appendix D) or allowed to be removed (Table 1), and in the latter case, it was significantly positively related to temporal variability in parasitism rates $\left(t=3.45, P_{\mathrm{MCMC}}\right.$ $=0.009$; Appendix C). Connectance was also negatively correlated with parasitoid and host species richness $(r=$ $-0.59, P<0.001$ and $r=-0.66, P<0.001$, respectively) but was not correlated with parasitoid redundancy $(r=$ $0.26, P=0.178$; Appendix B).

Spatial variability of parasitism rates (coefficient of variation of parasitism rates across plots) showed a marginally significant decrease with increasing parasitoid redundancy ( $t=-2.36, P=0.056$; Fig. $1 C)$, although low statistical power for this model $(\mathrm{df}=7)$ meant that this effect was only detected when allowing the removal of covariates in the model selection process (Table 1). Nevertheless, spatial variability of parasitism rates more than doubled (from 0.10 to 0.28 ) across the observed range of parasitoid redundancy (from 2.59 to 5.39), and redundancy was retained in the best-fitting model (Table $1)$.

\section{DisCUSSION}

Our study shows that empirical patterns of interactions among species can be linked to the mechanisms driving ecosystem functions and their stability, thereby extending them to large scales. We found that, at the community level, functional complementarity in host use among parasitoids was strongly associated with increased overall parasitism rates on hosts. Furthermore, the spatial stability (inverse of variability) of parasitism rates increased with parasitoid functional redundancy (i.e., host resource overlap), although no effect on temporal variability was detected.

Functional complementarity among parasitoids explained community-wide parasitism rates even after accounting for the effects of species richness, connectance, or taxonomic diversity. This suggests that, beyond the positive effect that parasitoid species richness can have on parasitism rates (Cardinale et al. 2003, Fenoglio et al. 2012), the pattern of resource use among species is also important for increasing attack rates, and it can easily be extracted from food-web data. Moreover, even though taxonomic diversity, which takes into account species relatedness, may be better than richness at representing community traits and functioning (Cadotte et al. 2008, Flynn et al. 2011, Srivastava et al. 2012), we found that taxonomic diversity did not affect parasitism rates, nor was it correlated with parasitoid complementarity. Hence, taxonomic diversity may not be an optimal measure of complementarity among predators, and preserving distantly related species per se might not be sufficient to secure ecosystem services (Macfadyen et al. 2012).

Previous experiments examining the effects of niche partitioning among a small number of parasitoid species (Finke and Snyder 2008) found that increasing the number of specialist species (i.e., those with higher functional complementarity) increased parasitism rates, but increasing the number of generalist species did not. The congruence of those results with our community- 
wide finding of functional complementarity increasing parasitism rates lends further support to the tenet that complementary resource use can be an important driver of ecosystem functioning in multitrophic systems (Finke and Snyder 2008, Gravel et al. 2011a, Striebel et al. 2012, Poisot et al. 2013) and that food-web structure is indeed closely linked to its functioning (Thompson et al. 2012).

In contrast to the importance of functional complementarity as a determinant of cumulative parasitism rates, we found no support for the hypothesis that parasitoid functional redundancy (the diversity of parasitoids attacking each host species) stabilizes temporal variation in parasitism rates in our study system. However, temporal variability in parasitism rates increased with food-web connectance. In a highly connected community, changes or fluctuations in the abundance of one species could affect several other species, and this effect could easily cascade to other members of the web (Melián and Bascompte 2002). Hence, highly connected food webs might have more variable parasitism rates, such as those observed for temporal variability in our study. This might also explain why in previous studies, increasing parasitoid diversity positively affected the temporal stability of parasitism rates (Tylianakis et al. 2006, Macfadyen et al. 2011), given that diversity and food-web connectance are usually negatively correlated (Riede et al. 2010).

The spatial variability of parasitism rates decreased with increasing parasitoid redundancy, contrary to what we observed for the temporal component. Food webs are connected with one another at landscape scales (Leibold et al. 2004), and their connection by dispersal determines the spatial insurance of ecosystem functions (Loreau et al. 2003, Gonzalez et al. 2009). The stabilizing effect of functional redundancy across native and plantation forests bears important consequences for biological control of forest pests. Maintenance of adjacent natural habitats, local introduction, or conservation of agents with generalist feeding strategies, or of multiple predators attacking each pest species would all potentially improve the stability of biological control across habitats.

Even though the importance of dispersal for spatial stability has been tested empirically (France and Duffy 2006, Symons and Arnott 2013), our study is, to our knowledge, the first empirical evidence that redundancy in resource use leads to spatial stability of consumption in food webs. Combined with the importance of network-wide trophic complementarity, we have demonstrated that known mechanisms through which diversity and redundancy affect ecosystem functioning and stability can be extrapolated to complex food webs. By placing a stronger conceptual emphasis on networks of interacting species, a food-web approach can be very useful for scaling up mechanisms to predict changes to ecosystem functioning.

\section{ACKNOWLEDGMENTS}

We thank J. Dugdale, J. Berry, and R. Schnitzler for help with insect identification, all the field and lab assistants who helped during the collections and rearing, and J. Ladley and D. Conder for logistical support. The Department of Conservation, Nelson Forests Ltd, Hancock Timber Resource Group, Merrill and Ring, and D. Bryant provided forest access. A. Varsani helped with molecular identifications. W. Godsoe, E. Espeland, the Tylianakis/Stouffer lab groups, and two anonymous reviewers provided helpful comments for improving the manuscript. G. Peralta is supported by the Marsden Fund (UOC-0802), C. Frost is supported by scholarships from the Natural Sciences and Engineering Research Council of Canada, Education New Zealand, and the University of Canterbury, R. K. Didham is funded by an Australian Research Council Future Fellowship, and J. M. Tylianakis is funded by a Rutherford Discovery Fellowship, administered by the Royal Society of New Zealand. This research was funded by the Marsden Fund (UOC-0802).

\section{Literature Cited}

Baayen, R. H. 2010. languageR: Data sets and functions with "analyzing linguistic data: a practical introduction to statistics.” $\mathrm{R}$ package version 1.0. http://CRAN.R-project. org/package=language $\mathrm{R}$

Balvanera, P., A. B. Pfisterer, N. Buchmann, J. S. He, T. Nakashizuka, D. Raffaelli, and B. Schmid. 2006. Quantifying the evidence for biodiversity effects on ecosystem functioning and services. Ecology Letters 9:1146-1156.

Bates, D., and M. Maechler. 2009. lme4: linear mixed-effects models using S4 classes. R package version 0.999375-32. http://CRAN.R-project.org/package=lme4

Bersier, L. F., C. Banasek-Richter, and M. F. Cattin. 2002. Quantitative descriptors of food-web matrices. Ecology 83: 2394-2407.

Bolker, B. M., M. E. Brooks, C. J. Clark, S. W. Geange, J. R. Poulsen, M. H. H. Stevens, and J. S. S. White. 2009. Generalized linear mixed models: a practical guide for ecology and evolution. Trends in Ecology and Evolution 24:127-135.

Cadotte, M. W., B. J. Cardinale, and T. H. Oakley. 2008. Evolutionary history and the effect of biodiversity on plant productivity. Proceedings of the National Academy of Sciences USA 105:17012-17017.

Cagnolo, L., A. Salvo, and G. Valladares. 2011. Network topology: patterns and mechanisms in plant-herbivore and host-parasitoid food webs. Journal of Animal Ecology 80: 342-351.

Cardinale, B. J., C. T. Harvey, K. Gross, and A. R. Ives. 2003. Biodiversity and biocontrol: emergent impacts of a multienemy assemblage on pest suppression and crop yield in an agroecosystem. Ecology Letters 6:857-865.

Cardinale, B. J., D. S. Srivastava, J. E. Duffy, J. P. Wright, A. L. Downing, M. Sankaran, and C. Jouseau. 2006. Effects of biodiversity on the functioning of trophic groups and ecosystems. Nature 443:989-992.

Chase, J. M., P. A. Abrams, J. P. Grover, S. Diehl, P. Chesson, R. D. Holt, S. A. Richards, R. M. Nisbet, and T. J. Case. 2002. The interaction between predation and competition: a review and synthesis. Ecology Letters 5:302-315.

Crowder, D. W., T. D. Northfield, M. R. Strand, and W. E. Snyder. 2010. Organic agriculture promotes evenness and natural pest control. Nature 446:109-113.

Daily, G. C., S. Polasky, J. Goldstein, P. M. Kareiva, H. A. Mooney, L. Pejchar, T. H. Ricketts, J. Salzman, and R. Shallenberger. 2009. Ecosystem services in decision making: time to deliver. Frontiers in Ecology and the Environment 7: 21-28.

Daily, G. C., et al. 2000. The value of nature and the nature of value. Science 289:395-396. 
Devoto, M., S. Bailey, P. Craze, and J. Memmott. 2012. Understanding and planning ecological restoration of plantpollinator networks. Ecology Letters 15:319-328.

Díaz, S., J. Fargione, F. S. Chapin, III, and D. Tilman. 2006. Biodiversity loss threatens human well-being. PLoS Biology 4:1300-1305.

Dormann, C. F., B. Fründ, N. Blüthgen, and B. Gruber. 2009. Indices, graphs and null models: analyzing bipartite ecological networks. Open Ecology Journal 2:7-24.

Dormann, C. F., B. Gruber, and J. Fründ. 2008. Introducing the bipartite package: analyzing ecological networks. R News 8:8-11.

Douglass, J. G., J. E. Duffy, and J. F. Bruno. 2008. Herbivore and predator diversity interactively affect ecosystem properties in an experimental marine community. Ecology Letters 11:598-608

Duffy, J. E. 2002. Biodiversity and ecosystem function: the consumer connection. Oikos 99:201-219.

Fenoglio, M. S., D. Srivastava, G. Valladares, L. Cagnolo, and A. Salvo. 2012. Forest fragmentation reduces parasitism via species loss at multiple trophic levels. Ecology 93:2407-2420.

Finke, D. L., and W. E. Snyder. 2008. Niche partitioning increases resource exploitation by diverse communities. Science 231:1488-1490.

Flynn, D. F. B., N. Mirotchnick, M. Jain, M. I. Palmer, and S. Naeem. 2011. Functional and phylogenetic diversity as predictors of biodiversity-ecosystem-function relationships. Ecology 92:1573-1581.

France, K. E., and J. E. Duffy. 2006. Diversity and dispersal interactively affect predictability of ecosystem function. Nature 441:1139-1143.

Gonzalez, A., N. Mouquet, and M. Loreau. 2009. Biodiversity as spatial insurance: the effects of habitat fragmentation and dispersal on ecosystem functioning. Pages 134-146 in S. Naeem, D. Bunker, A. Hector, M. Loreau, and C. Perrings, editors. Biodiversity, ecosystem functioning, and human wellbeing. Oxford University Press, New York, New York, USA.

Gravel, D., T. Bell, C. Barbera, T. Bouvier, T. Pommier, P. Venail, and N. Mouquet. 2011a. Experimental niche evolution alters the strength of the diversity-productivity relationship. Nature 469:89-92.

Gravel, D., E. Canard, F. Guichard, and N. Mouquet. $2011 b$. Persistence increases with diversity and connectance in trophic metacommunities. PLoS ONE 6:e19374.

Helmus, M. R., T. J. Bland, C. K. Williams, and A. R. Ives. 2007. Phylogenetic measures of biodiversity. American Naturalist 169:E68-E83.

Hillebrand, H., and B. J. Cardinale. 2004. Consumer effects decline with prey diversity. Ecology Letters 2004:192-201.

Hooper, D. U., et al. 2005. Effects of biodiversity on ecosystem functioning: a consensus of current knowledge. Ecological Monographs 75:3-35.

Ives, A. R., B. J. Cardinale, and W. E. Snyder. 2005. A synthesis of subdisciplines: predator-prey interactions, and biodiversity and ecosystem functioning. Ecology Letters 8: $102-116$.

Kembel, S. W., P. D. Cowan, M. R. Helmus, W. K. Cornwell, H. Morlon, D. D. Ackerly, S. P. Blomberg, and C. O. Webb. 2010. Picante: $\mathrm{R}$ tools for integrating phylogenies and ecology. Bioinformatics 26:1463-1464.

Kozak, K. H., and J. J. Wiens. 2010. Accelerated rates of climatic-niche evolution underlie rapid species diversification. Ecology Letters 13:1378-1389.

Leibold, M. A., et al. 2004. The metacommunity concept: a framework for multi-scale community ecology. Ecology Letters 7:601-613.

Loreau, M., N. Mouquet, and A. Gonzalez. 2003. Biodiversity as spatial insurance in heterogeneous landscapes. Proceedings of the National Academy of Sciences USA 100:12765-12770.
Macfadyen, S., P. Craze, A. Polaszek, K. van Achterberg, and J. Memmott. 2011. Parasitoid diversity reduces the variability in pest control services across time on farms. Proceedings of the Royal Society B 278:3387-3394.

Macfadyen, S., S. A. Cunningham, A. C. Costamagna, and N. A. Schellhorn. 2012. Managing ecosystem services and biodiversity conservation in agricultural landscapes: are the solutions the same? Journal of Applied Ecology 49:690-694.

Macfadyen, S., R. Gibson, A. Polaszek, R. J. Morris, P. G. Craze, R. Planque, W. O. C. Symondson, and J. Memmott. 2009. Do differences in food web structure between organic and conventional farms affect the ecosystem service of pest control? Ecology Letters 12:229-238.

Maherali, H., and J. N. Klironomos. 2007. Influence of phylogeny on fungal community assembly and ecosystem functioning. Science 316:1746-1748.

Marino, P. C., and D. A. Landis. 1996. Effect of landscape structure on parasitoid diversity and parasitism in agroecosystems. Ecological Applications 6:276-284.

May, R. M. 1973. Stability versus complexity in multispecies models. Pages 37-78 in R. M. May, editor. Stability and complexity in model ecosystems. Princeton University Press, Princeton, New Jersey, USA.

Melián, C. J., and J. Bascompte. 2002. Complex networks: two ways to be robust? Ecology Letters 5:705-708.

Menalled, F. D., P. C. Marino, S. H. Gage, and D. A. Landis. 1999. Does agricultural landscape structure affect parasitism and parasitoid diversity? Ecological Applications 9:634-641.

Mills, N. J., and E. Wajnberg. 2008. Optimal foraging behaviour and efficient biological control methods. Blackwell Publishing Ltd, Oxford, UK.

Montoya, J. M., S. L. Pimm, and R. V. Sole. 2006. Ecological networks and their fragility. Nature 442:259-264.

Montoya, J. M., M. A. Rodriguez, and B. A. Hawkins. 2003. Food web complexity and higher-level ecosystem services. Ecology Letters 6:587-593.

Naeem, S., and S. Li. 1997. Biodiversity enhances ecosystem reliability. Nature 390:507-509.

Pennisi, E. 2010. The little wasp that could. Science 327.

Poisot, T., N. Mouquet, and D. Gravel. 2013. Trophic complementarity drives the biodiversity-ecosystem functioning relationship in food webs. Ecology Letters 16:853-861.

Poulin, R., and D. Mouillot. 2003. Parasite specialization from a phylogenetic perspective: a new index of host specificity. Parasitology 126:473-480.

R Development Core Team. 2012. R: a language and environment for statistical computing. R Foundation for Statistical Computing, Vienna, Austria. www.r-project.org

Richards, A. E., and S. Schmidt. 2010. Complementary resource use by tree species in a rain forest tree plantation. Ecological Applications 20:1237-1254.

Riede, J. O., B. C. Rall, C. Banasek-Richter, S. A. Navarrete, E. A. Wieters, M. C. Emmerson, U. Jacob, and U. Brose. 2010. Scaling of food-web properties with diversity and complexity across ecosystems. Pages 139-170 in G. Woodward, editor. Advances in ecological research. Volume 42. Academic Press, Burlington, Massachusetts, USA.

Rooney, N., and K. S. McCann. 2012. Integrating food web diversity, structure and stability. Trends in Ecology and Evolution 27:40-46.

Sekercioglu, S. H. 2010. Ecosystem functioning and services. Pages 45-72 in N. S. Sodhi and P. R. Ehrlich, editors. Conservation biology for all: ecosystem functions and services. Oxford University Press, New York, New York, USA.

Snyder, W. E., G. B. Snyder, D. L. Finke, and C. S. Straub. 2006. Predator biodiversity strengthens herbivore suppression. Ecology Letters 9:789-796.

Srivastava, D. S., M. W. Cadotte, A. M. MacDondald, R. G. Marushia, and N. Mirotchnick. 2012. Phylogenetic diversity and the functioning of ecosystems. Ecology Letters 15:637-648. 
Straub, C. S., D. L. Finke, and W. E. Snyder. 2008. Are the conservation of natural enemy diversity and biological control compatible goals? Biological Control 45:225-237.

Striebel, M., G. Singer, H. Stibor, and T. Andersen. 2012. "Trophic overyielding": phytoplankton diversity promotes zooplankton productivity. Ecology 93:2719-2727.

Symons, C. C., and S. E. Arnott. 2013. Regional zooplankton dispersal provides spatial insurance for ecosystem function. Global Change Biology 19:1610-1619.

Thébault, E., and M. Loreau. 2003. Food-web constraints on biodiversity-ecosystem functioning relationships. Proceedings of the National Academy of Sciences USA 100:1494914954.

Thompson, R. M., et al. 2012. Food webs: reconciling the structure and function of biodiversity. Trends in Ecology and Evolution 27:689-697.

Tylianakis, J. M., and A. Binzer. In press. Effects of global environmental changes on parasitoid-host food webs and biological control. Biological Control. http://dx.doi.org/10. 1016/j.biocontrol.2013.10.003

Tylianakis, J. M., T. A. Rand, A. Kahmen, A. M. Klein, N. Buchmann, J. Perner, and T. Tscharntke. 2008. Resource heterogeneity moderates the biodiversity-function relationship in real world ecosystems. PLoS Biology 6:947-956.
Tylianakis, J. M., T. Tscharntke, and A. M. Klein. 2006. Diversity, ecosystem function, and stability of parasitoid host interactions across a tropical habitat gradient. Ecology 87: 3047-3057.

Tylianakis, J. M., T. Tscharntke, and O. T. Lewis. 2007. Habitat modification alters the structure of tropical hostparasitoid food webs. Nature 445:202-205.

Walker, B. H. 1992. Biodiversity and ecological redundancy. Conservation Biology 6:18-23.

Weiblen, G. D., C. O. Webb, V. Novotny, Y. Basset, and S. E. Miller. 2006. Phylogenetic dispersion of host use in a tropical insect herbivore community. Ecology 87:S62-S75.

Wilby, A., and K. H. Orwin. 2013. Herbivore species richness, composition and community structure mediate predator richness effects and top-down control of herbivore biomass. Oecologia 172:1167-1177.

Yachi, S., and M. Loreau. 1999. Biodiversity and ecosystem productivity in a fluctuating environment: the insurance hypothesis. Proceedings of the National Academy of Sciences USA 96:1463-1468.

Yachi, S., and M. Loreau. 2007. Does complementarity resource use enhance ecosystem functioning? A model of light competition in plant communities. Ecology Letters 10: $54-62$.

\section{Supplemental Material}

\section{Appendix A}

Study region, sampling, molecular identification technique, and species collected (Ecological Archives E095-166-A1).

\section{Appendix B}

Correlation between predictor variables and covariates (Ecological Archives E095-166-A2).

\section{Appendix C}

Relationship between food-web connectance and temporal variability of parasitism rates (Ecological Archives E095-166-A3).

\section{Appendix D}

Coefficients for best-fitting model without covariate removal (Ecological Archives E095-166-A4). 